\title{
Preparation and characterization of an attenuated porcine epidemic diarrhea virus strain by serial passaging
}

\author{
Tao Jie ${ }^{1,2,3} \cdot$ Li Benqiang $^{1,2,3} \cdot$ Cheng Jinghua ${ }^{1,2,3} \cdot$ Shi Ying ${ }^{1,2,3} \cdot$ Liu Huili $^{1,2,3}$
}

Received: 10 December 2017 / Accepted: 12 May 2018 / Published online: 31 July 2018

(c) Springer-Verlag GmbH Austria, part of Springer Nature 2018

\begin{abstract}

\begin{tabular}{ll}
\multicolumn{2}{l}{ Abbreviations } \\
PEDV & Porcine epidemic diarrhea virus \\
TGEV & Transmissible gastroenteritis virus \\
ORF & Open reading frame \\
S & Spike \\
N & Nucleocapsid \\
M & Membrane \\
Dpi & Days of postinfection
\end{tabular}
\end{abstract}

Porcine epidemic diarrhea virus (PEDV) is prevalent in most parts of the world. Owing to its antigenic variation, prevention of the diseases caused by this virus is difficult. In this study, two PEDV isolates with similar growth kinetics were successfully propagated in Vero cells. Complete genome sequence analysis showed that they have a 49nt deletion in the ORF3 gene and were classified into Group 1, the same group that includes the classical CV777 strain. Recombination analysis revealed that the event had occurred in the ORF1a gene, at 3596-6819 nt, among the two PEDV isolates and the CV777 and DR13 strains. During their continuous propagation, 14 nonsynonymous mutations occurred in the spike (S) gene of strain JS-2/2014 between generations G5 and G90, but there were no changes between G90 and G100. We assumed that strain JS-2/2014 might be attenuated by the 90th generation. Piglets orally fed with JS-2/2014 G90 showed no clinical symptoms, and no virus was detected in the feces and nasal fluid. In conclusion, JS-2/2014 was successfully identified by screening, was attenuated after propagation in Vero cells, and may serve as a candidate virus for vaccine preparations.

\section{Introduction}

Porcine epidemic diarrhea virus (PEDV) is the causative agent of porcine epidemic diarrhea, an enteric disease characterized by acute watery diarrhea, dehydration, and

Handling Editor: Sheela Ramamoorthy.

Liu Huili

huilil@163.com

1 Institute of Animal Science and Veterinary Medicine, Shanghai Academy of Agricultural Sciences, Shanghai 201106, China

2 Shanghai Key Laboratory of Agricultural Genetic Breeding, Shanghai 201106, China

3 Shanghai Engineering Research Center of Pig Breeding, Shanghai 201302, China vomiting and which affects pigs of all ages but has a high mortality rate among neonatal piglets [1]. PEDV was first described in England in 1971, and until the end of the 1990s, sporadic cases were reported throughout Europe [2-5]. Since October 2010, severe PEDV outbreaks have occurred in the domestic pig population in China and caused enormous economic losses [6-9].

PEDV vaccines based on the classical PEDV strain CV777, namely the inactivated bivalent transmissible gastroenteritis virus (TGEV) \& PEDV vaccine (1999 - present) and the attenuated bivalent TGEV \& PEDV vaccine (2003-2006) were widely used in China and played an important role in controlling the disease. Nonetheless, an outbreak of diarrheal disease in 2010 and the high mortality rate among neonatal piglets indicated that the epidemic virus may have changed and escaped the immune response specific to the vaccine [10]. It was subsequently confirmed that variants were responsible for the large-scale outbreak of the disease [11].

The spike (S) protein of coronaviruses performs an important function: binding to cellular receptors and initiating the infection. It also induces neutralizing antibodies in vivo. Mutations, including deletions and/or insertions, in the $\mathrm{S}$ protein may change the pathogenicity and tissue tropism of coronaviruses; and this mechanism may be the main reason for the virus escaping the immune response generated by vaccination [12]. Therefore, it is necessary to analyze the 
sequence and characteristics of the prevalent PEDV strains for further exploration of these pathogenic mechanisms.

In this study, we first determined the complete genome sequences and biological characteristics of PEDV strains that have emerged in domestic pigs. Subsequently, the genetic relationship between these and other PEDV strains was analyzed. Furthermore, we cultured the virus and screened for attenuated PEDV strains by analyzing the genovariations that occurred during passaging - confirming the results in animal experiments. Our results provide useful insights into the preparation of an effective and safe PEDV vaccine.

\section{Materials and methods}

\section{Ethics statement}

All animal experiments were conducted under the guidance of the Institutional Animal Care and Use Committee at Centers for Disease Control and Prevention (CDC) and the Laboratory Animal Care International accredited facility.

\section{Origin of the PEDV isolates}

Strains JSLS-1/2014 and JS-2/2014 were isolated from the intestinal tissues of piglets with watery diarrhea and were sent to our laboratory for virus detection. Sows were all inoculated with the bivalent killed or attenuated vaccines against TGEV \& PEDV. As many as 50\% of piglets died within 2 days of birth. Pigs from another farm died at $\sim 1$ month of age following the development of watery diarrhea in the clinic.

\section{Preparation of a PEDV inoculum for Vero cells}

PEDV-positive fecal samples were diluted 10-fold in PBS and then vortexed briefly, followed by centrifugation at $10,000 \times g$ for $10 \mathrm{~min}$. The supernatants were passed through $0.22 \mu \mathrm{m}$ syringe filters and then inoculated onto confluent Vero cells. After adsorption at $37^{\circ} \mathrm{C}$ for $1 \mathrm{~h}$, the cells were incubated in Dulbecco's modified Eagle's medium (DMEM). The PEDV strains were identified by RT-PCR and an indirect immunofluorescence assay (IFA).

For the IFA, the Vero cells were fixed with $70 \%$ ethanol and incubated with an anti-PEDV monoclonal antibody for $1 \mathrm{~h}$, followed by incubation with a fluorescein isothiocyanate (FITC)-labeled goat anti-mouse IgG antibody. Finally, cell staining was examined under a fluorescence microscope.

\section{Plaque staining and purification}

The PEDV isolates were purified by a plaque method in Vero cells. In brief, confluent cell monolayers in 6-well plates were infected with 10 -fold dilutions of each virus isolate (to a total volume of $0.4 \mathrm{~mL}$ of PBS) for $1 \mathrm{~h}$ at $37^{\circ} \mathrm{C}$. The cells were washed twice with PBS and overlaid with modified Eagle's medium containing $0.9 \%$ of agar. The plates were then incubated at $37{ }^{\circ} \mathrm{C}$ and $5 \% \mathrm{CO}_{2}$. After 5 days of incubation, the cells were stained with $0.1 \%$ crystal violet [13].

\section{Full-length genome sequencing and phylogenetic analysis}

Viral RNA was extracted from a $250 \mu \mathrm{L}$ sample using the TRIzol Reagent (Takara, Shiga, Japan). The PEDV cDNA was synthesized by means of the PrimeScript High Fidelity RT-PCR Kit (Takara). Full-length PEDV genome amplification was performed with primers described previously [14]. The $5^{\prime}$ and $3^{\prime}$ end sequences were determined with the $5^{\prime}$ and 3' RACE kit (Takara). For each amplicon, more than three independent clones were sequenced to determine the consensus sequence of a given genomic region.

The ClustalX (ver.1.81) software was used to align the full-length genome sequences. Phylogenetic analyses based on the $S$ and open reading frame 3 (ORF3) genes or the entire genome were performed by the maximum-likelihood method with the general time-reversible nucleotide substitution model, where 1000 bootstrap replicates were implemented in the MEGA6.0 software [15].

\section{Growth kinetics}

Growth kinetics of the PEDV strains were measured in Vero cells and compared. The viruses were added at a multiplicity of infection (MOI) of 1 to Vero cells in a 24-well plate. After $1 \mathrm{~h}$, the cell medium was replaced with fresh DMEM containing $2.5 \mu \mathrm{g} / \mathrm{mL}$ trypsin. Culture samples collected at 12 , $24,48,60,72$, and $96 \mathrm{~h}$ postinfection were freeze-thawed twice and then centrifuged at $12,000 \times g$ for $10 \mathrm{~min}$ at $4{ }^{\circ} \mathrm{C}$. The supernatants were collected and analyzed for the virus titer $\left(50 \%\right.$ tissue culture infectious dose $\left[\mathrm{TCID}_{50}\right] / \mathrm{mL}$ ) following the Reed-Muench method [16].

\section{Animal experiments}

To evaluate the pathogenicity of a PEDV strain, we analyzed and purchased PEDV-negative pregnant sows. Negativity for known enteric pathogens was confirmed by quantitative RT-PCR. Pigs were orally inoculated with the JS-2/2014 strain (different passages), 1-day-old neonatal piglets were given access to milk and water ad libitum (three piglets per group). The virus inoculum was $10^{6} \mathrm{TCID}_{50}$ per animal. Clinical symptoms, survival, and virus shedding (daily rectal and throat swabs) were then examined. Virus shedding was quantified by RT-PCR with the following primers, PEDV-F: 5'-TTCCCGTTGATGAGGTGAT-3', PEDV-R: 5'-AAGCAT 
TGACTGAACGACC-3'. Serum and feces samples were collected from each piglet weekly and tested for IgG and IgA antibodies by ELISA.

\section{Results}

\section{Isolation of PEDV and its biological characteristics}

We isolated six PEDV strains in this study. Due to their high identity, only two strains - JSLS-1/2014 and JS-2/2014 were chosen for genome analysis. They proliferated successfully in Vero cells without trypsin (Fig. 1a, b) and induced extensive cell death (lysis) without cell fusion (Fig. 1c). The plaque assay confirmed that they had a similar morphology (Fig. 1d). Furthermore, their growth characteristics indicated that they had similar growth kinetics, with replication peaking $72 \mathrm{~h}$ after infection (Fig. 1e).

\section{Full-length genome characterization}

The genomes of the PEDV isolates were sequenced after the 5 th passage. The details for each gene are listed in Table 1. Complete sequence homology analysis of the PEDV isolates and other PEDV reference strains revealed that they shared high identity $(99.0 \%-99.9 \%)$ with domestic strains OH851 and FL2013. The phylogenetic tree based on the complete genomic sequences indicated that JSLS-1/2014 and JS-2/2014 isolates clustered in the same group together with classical strains CV777 and DR13 (Fig. 2a).

Next, analysis of the $N$-glycosylation sites within the $\mathrm{S}$, membrane $(\mathrm{M})$, and nucleocapsid $(\mathrm{N})$ proteins indicated
Fig. 1 Cellular characteristics of the two PEDV isolates. PEDVpositive fecal samples were inoculated onto Vero cells in continuous passage culture. (a) The $3^{\text {rd }}$-passage viruses yielded positive bands of $\sim 550 \mathrm{bp}$ in RT-PCR analysis with primers specific to the $M$ gene of PEDV. (b) Indirect immunofluorescence assay with DAPI confirmed that PEDV strains JSLS-1/2014 and JS-2/2014 could be recognized by the antiPEDV monoclonal antibody. (c) After six blind passages, Vero cells infected with the PEDV isolates showed obvious cytopathic effects with widespread death and shedding of the virus. (d) PEDV virus particles were purified by a plaque method, with picking of a single clone each time. The results showed that the plaque morphological features of strains JSLS-1/2014 and JS-2/2014 were similar. (e) Growth kinetics assays indicated that both strains could replicate effectively in Vero cells with similar characteristics (a)



(b)
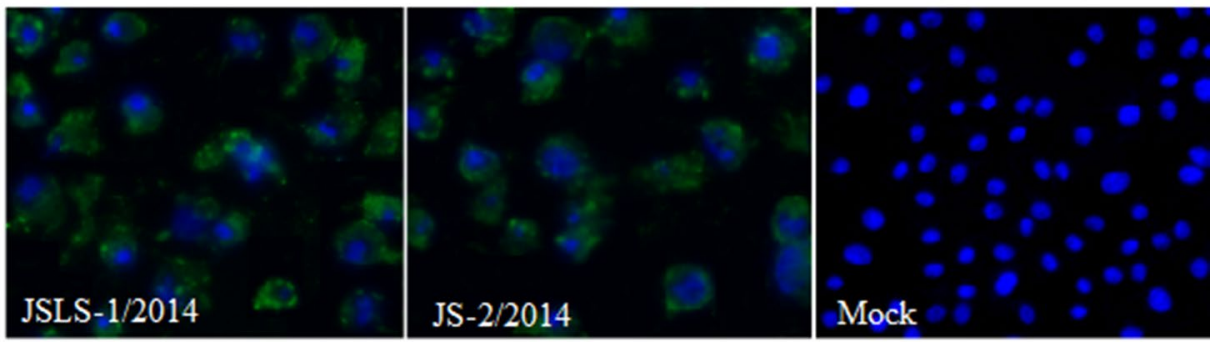

(c)

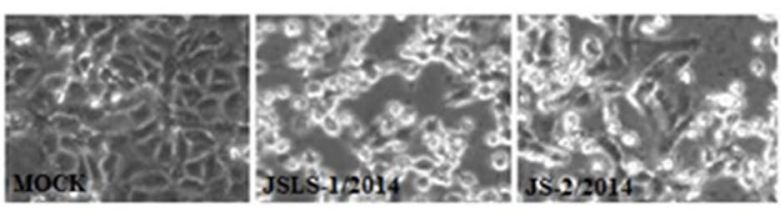

(d)

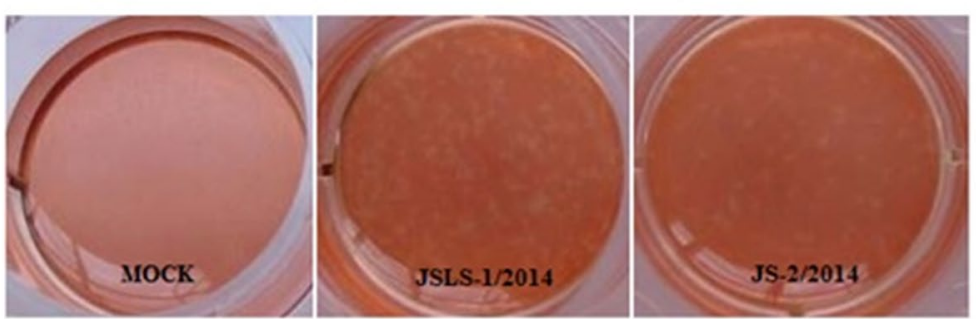

(e)

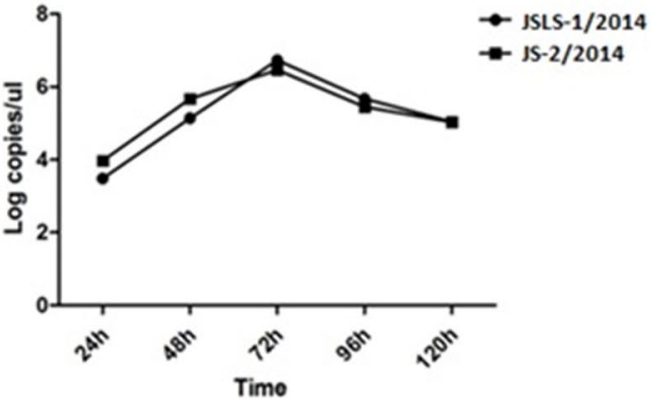


Table 1 Gene information for the porcine epidemic diarrhea virus isolates

\begin{tabular}{llllllllll}
\hline Strains & \multicolumn{2}{l}{ Location of the genes } & & & & & \\
& \cline { 2 - 9 } & 5'-UTR & ORF1a & ORF1b & S & ORF3 & E & M & N \\
\hline JSLS-1/2014 & $1-291$ & $292-12576$ & $12576-20540$ & $20537-24685$ & $24685-24960$ & $25291-25500$ & $25508-26188$ & $26200-27525$ & $27526-27869$ \\
JS-2/2014 & $1-292$ & $293-12574$ & $12574-20610$ & $20607-24755$ & $24577-25030$ & $25361-25570$ & $25578-26258$ & $26270-27595$ & $27596-27939$ \\
\hline
\end{tabular}

(a)

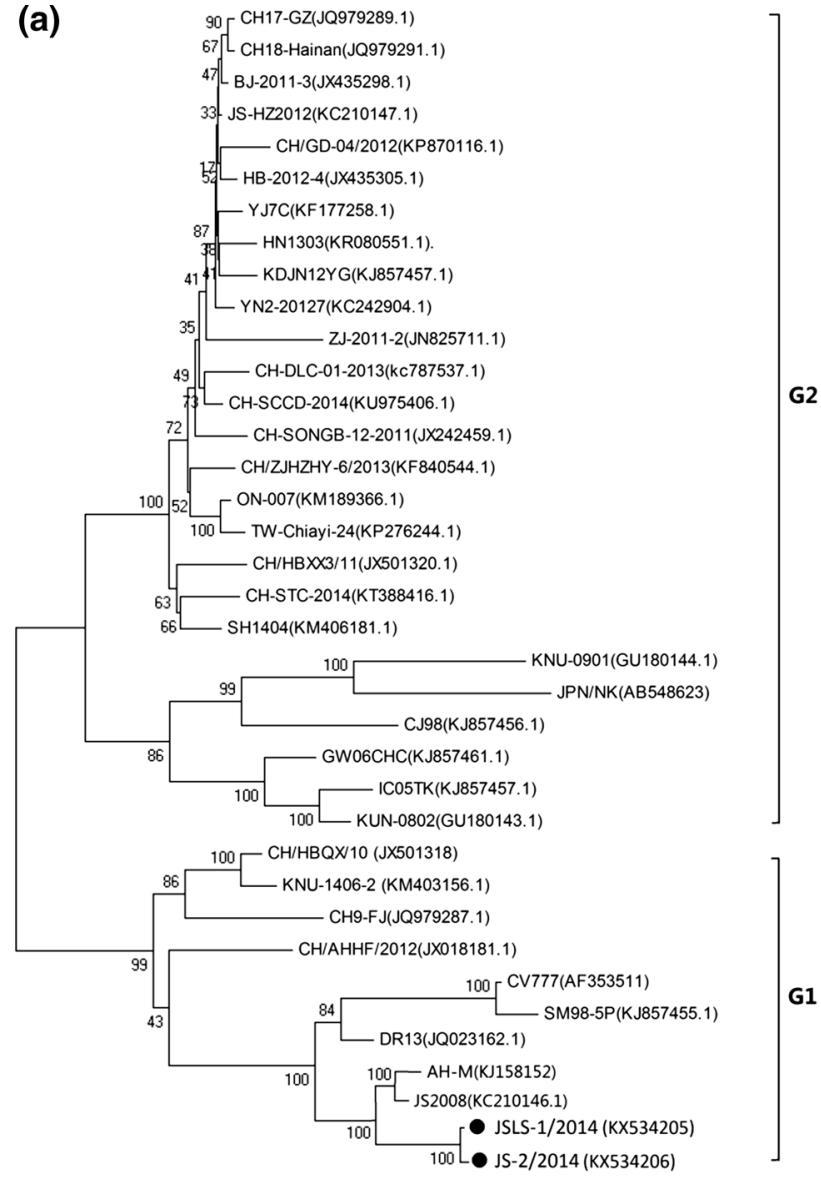

$\stackrel{\longmapsto}{\triangleright .005}$

Fig. 2 Phylogenetic analysis based on the nucleotide sequences of the complete genome (a) and $S$ gene (b) of our PEDV strains along with reference PEDV strains. The tree was constructed by the neighbor-joining method in the MEGA6.0 software. The numbers at the

that JSLS-1/2014 and JS-2/2014 had the same number of $\mathrm{N}$-glycosylation sites in the $\mathrm{S}$ protein; one less than the number in the attenuated DR13 strain (Table 2). In the M protein, our two PEDV strains and attenuated DR13 had three $N$-glycosylation sites, whereas CV777 had one more site. In the $\mathrm{N}$ protein, all the strains had six $\mathrm{N}$-glycosylation sites, except for JSLS-1/2014 which had one less.



branches are bootstrap values (\%) following 1000 replicates. A dot has been added to the front of the two PEDV strains isolated in this study

Table $2 N$-glycosylation sites in the PEDV strain $S, M$ and $N$ genes

\begin{tabular}{lllll}
\hline Genes & N-Glyc & & & \\
\cline { 2 - 5 } & JSLS-1/2014 & JS-2/2014 & $\begin{array}{l}\text { attenuated } \\
\text { DR13 }\end{array}$ & CV777 \\
\hline S & 27 & 27 & 28 & 29 \\
M & 3 & 3 & 3 & 4 \\
N & 5 & 6 & 6 & 6 \\
\hline
\end{tabular}




\section{Phylogenetic and sequence analyses of genes $S$, $O R F 3, M$, and $N$}

The full $S$ sequences of our PEDV isolates and of 33 reference strains from Korea, Germany, the US, and China were compared. The phylogenetic tree, constructed by the neighbor-joining method, showed that the two PEDV isolates from this study clustered within the same group as the classical strains, and genetically distant from the Chinese PEDV strains isolated during 2011-2014 (Fig. 2b). Further comparison indicated that the $S 1$ genes from our two PEDV isolates were conserved, with amino acid identities of $99.4-99.8 \%$. Nevertheless, they had obvious variation, relative to the domestic PEDV strains isolated before 2014, with amino acid identities of 89.6-91.3\%.

Analysis of the ORF3 genes revealed $99.2 \%$ homology in the encoded amino acid sequence between JSLS$1 / 2014$ and JS-2/2014 isolates, whereas with other strains it reached 100\% (e.g., $\mathrm{AH} / \mathrm{HF} / 2015, \mathrm{HeB} / 2015 / 121$, and $\mathrm{HLJ} /$ $\mathrm{QQHR} / 2015)$. This meant that there is a certain degree of variation between our two PEDV isolates even though their identity is high. In addition, a 49nt sequence was found to be deleted in the ORF3 gene in our two PEDV isolates - a feature also found in the attenuated strains DR13, HLJBY, AH-M, and JS2008. This deletion is a typical characteristic of cell-adapted PEDV strains [17]. On the other hand, the ORF3 gene of other domestic PEDV strains isolated in recent years is intact in the same way as CV777. It was therefore speculated that the pathogenicity of our PEDV isolates was likely attenuated.

The M protein is one of the important proteins of PEDV for activating host immunity. Amino acid comparison indicated that there were only three amino acid mutations between our PEDV isolates, CV777, and the attenuated DR13: at amino acid positions 5, 56, and 167 (data not shown). The sequence between amino acid residues 1 and 50 is a potential epitope, and the fact that most of the site mutations are located in this area implies antigenic diversity.

By analyzing the amino acid sequences of the $\mathrm{N}$ protein, we found great differences among the PEDV isolates, CV777, and attenuated DR13. Compared with CV777, strain JS-2/2014 has 14 amino acid mutations (data not shown). Compared with attenuated DR13, strain JS-2/2014 has five amino acid mutations, whereas JSLS-1/2014 has no changes.

\section{Recombination analysis}

To determine the involvement of recombination events in the evolution of the isolates, we performed recombination analysis, using the RDP4 software, to compare our two isolates with representative Chinese historical PEDV strains from different clusters in the phylogenetic tree. The findings indicated recombination among the two PEDV isolates and CV777 and DR13 had occurred in the ORF1a gene at site 3596-6819 (nt positions; Fig. 3a), with a higher recombination probability being detected for CV777 (Fig. 3b). The

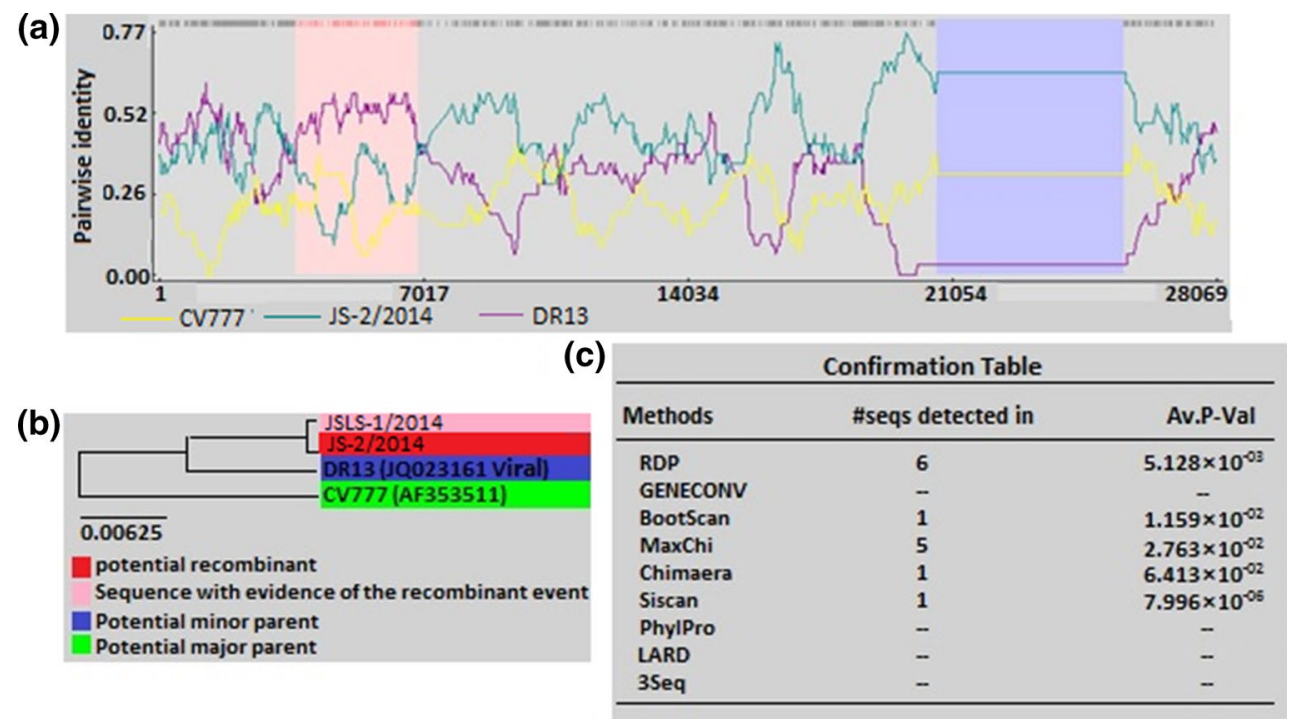

Fig. 3 Recombination analysis of the PEDV isolates. Detection of potential recombination events in the two PEDV strains in the RDP4 software. (a) Four major recombination breakpoints were located, respectively, in genes ORF1a (nt3596 and nt6819), S (nt20743), and $M$ (nt25758). The analysis was performed with an F84 distance model, a window size of $1000 \mathrm{bp}$, and a step size of $200 \mathrm{bp}$. (b) Evolutionary relations among the two PEDV strains and the putative parental strains on the basis of full-length genomes. Phylogenetic trees were generated by the distance-based neighbor-joining method in the RDP4 program. (c) Scores of the recombination events detected by the nine methods in RDP4 
average $\mathrm{P}$ values for JSLS-1/2014 and JS-2/2014 were 3.677 $\times 10^{-3}$ and $9.426 \times 10^{-3}$, respectively (Fig. $3 \mathrm{c}$ ).

\section{Genovariation during cell propagation}

It has been reported that the PEDV $S$ gene demonstrates the biggest variability during evolution, prompting us to explore its key genovariation during cell propagation. These data will provide important information for researchers regarding cellular adaptation, virus attenuation, and pathogenicity.

Strain JS-2/2014 was serially passaged in Vero cells, and the 5th (G5), 20th (G20), 50th (G50), and 90th (G90) generations of the virus were selected and sequenced. Comparison of the amino acid sequences revealed that there were 14 missense mutations in the $S$ gene (Table 3). Three amino acid residues (at sites 288, 330, and 973) were mutated by G20, six (at sites 360, 784, 816, 901, 983, and 1033) more were mutated at G50, and five more (at sites 15, 56, 132, 1204 , and 1308) were mutated by G90. It is worth noting that the amino acid variations at passages G20 and G50 also participated in the DR13 attenuation process. Nevertheless, the five other amino acid mutations were not seen in other PEDV strains and may be specific to strain JS-2/2014.

\section{The JS-2/2014 G90 strain was not pathogenic to piglets munity}

Strains JS-2/2014 G20, JS-2/2014 G60, and JS-2/2014 G90 at a titer of $10^{6} \mathrm{TCID}_{50}$ were orally fed to PEDV antibodynegative weaned piglets. After the virus challenge, the piglets fed with strain JS-2/2014 G90 appeared asymptomatic, and no virus was detected in the feces and nasal fluid. By contrast, piglets infected with the JS-2/2014 G20 strain developed watery diarrhea and died at 5 days post-infection (dpi) (Fig. 4a). In addition, the piglets infected with the JS-2/2014 G60 strain did not develop diarrhea but had to be detoxified for a period (Fig. 4b). We found that piglets inoculated with JS-2/2014 G60 grew slowly before 6 dpi, whereas the weight gain of piglets inoculated with JS-2/2014 G90 was normal relative to that of control piglets (Fig. 4c). Furthermore, infection with JS-2/2014 G90 activated strong IgG and IgA antibody responses (Fig. 4d). These results confirmed that the JS-2/2014 G90 strain was attenuated, and this change was attributed to its repeated propagation in tissue culture.

\section{Discussion}

Porcine diarrheal disease has become epidemic in China in recent years, causing huge financial losses in the pig industry [18]. PEDV, TGEV, and porcine rotavirus (PoRV) are the three main pathogens of this disease $[19,20]$. To trace the epidemiology of the recent outbreaks, porcine fecal samples were collected; our results revealing that PEDV had a higher infection rate than both TGEV and PoRV. This result is consistent with data from other reports [21, 22].

To explore the evolution and pathogenicity of this virus, we next identified two PEDV variants in porcine fecal samples from the Shanghai and Jiangsu provinces. Of note, unlike the PEDV variants isolated in China in recent years, our two isolates could propagate in Vero cells successfully without trypsin. Nevertheless, most of the PEDV variants isolated in recent years can proliferate in mammalian cells successfully only in the presence of trypsin [23]. It is unknown whether this phenomenon is related to the $S$ gene, which is important for cell adaptation [24], or to the ORF3 gene, which is a recognized marker for attenuated and virulent strains [25].

Our sequence analysis revealed that these two isolates clustered in the same group as classical strain CV777, and all had a 49nt deletion in the $O R F 3$ gene. Sun et al. [14] reported that all newly isolated strains had intact ORFs that could yield translation of a 224 aa protein. These studies suggest that this area of the genome may be involved in determining cell tropism and pathogenicity of the virus [26]. Park et al. [27] analyzed a cell culture-adapted PEDV (passage 100) strain with a smaller $O R F 3$ gene and found it to have lower virulence relative to the wild-type virus. This may mean that the pathogenicity of our two PEDV isolates is different from that of the PEDV variants isolated in recent years.

The S protein of PEDV has always been used as a marker of viral variation. Under the pressure of herd immunity, the $S$ gene of PEDV mutates frequently, with some of the missense mutations altering viral antigenicity to aid in the
Table 3 Genovariation of the $S$ gene in the JS-2/2014 strain during propagation

\begin{tabular}{|c|c|c|c|c|c|c|c|c|c|c|c|c|c|c|c|}
\hline \multirow{2}{*}{\multicolumn{2}{|c|}{$\begin{array}{l}\text { Strains of different } \\
\text { passage number }\end{array}$}} & \multicolumn{14}{|c|}{ Location of the amino acid } \\
\hline & & 15 & 56 & 132 & 288 & 330 & 360 & 784 & 816 & 901 & 973 & 983 & 1033 & 1204 & 1308 \\
\hline \multirow[t]{5}{*}{ JS-2/2014 } & G5 & $S$ & G & I & $\mathrm{L}$ & $S$ & $\mathrm{~L}$ & $\mathrm{~T}$ & $\mathrm{~F}$ & $\mathrm{R}$ & $\mathrm{F}$ & $\mathrm{Y}$ & $\mathrm{K}$ & $\mathrm{H}$ & Q \\
\hline & G20 & $S$ & $\mathrm{G}$ & I & $\mathrm{W}$ & $\mathrm{F}$ & $\mathrm{L}$ & $\mathrm{T}$ & $\mathrm{F}$ & $\mathrm{R}$ & $\mathrm{V}$ & $\mathrm{Y}$ & $\mathrm{K}$ & $\mathrm{H}$ & Q \\
\hline & G50 & $\mathrm{S}$ & G & I & $\mathrm{W}$ & $\mathrm{F}$ & $\mathrm{K}$ & M & V & $\mathrm{G}$ & $\mathrm{V}$ & $\mathrm{H}$ & $\mathrm{N}$ & $\mathrm{H}$ & Q \\
\hline & G90 & $\mathrm{L}$ & M & $\mathrm{N}$ & $\mathrm{W}$ & $\mathrm{F}$ & $\mathrm{K}$ & M & $\mathrm{V}$ & $\mathrm{G}$ & $\mathrm{V}$ & $\mathrm{H}$ & $\mathrm{N}$ & $\mathrm{Y}$ & $\mathrm{R}$ \\
\hline & G100 & $\mathrm{L}$ & $\mathrm{M}$ & $\mathrm{N}$ & W & $\mathrm{F}$ & K & M & V & G & V & $\mathrm{H}$ & $\mathrm{N}$ & Y & $\mathrm{R}$ \\
\hline
\end{tabular}


(a)

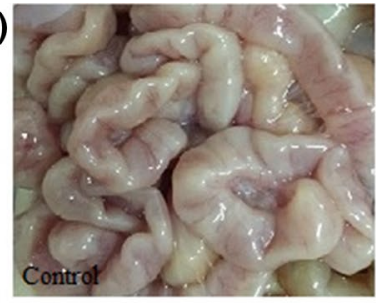

(b)

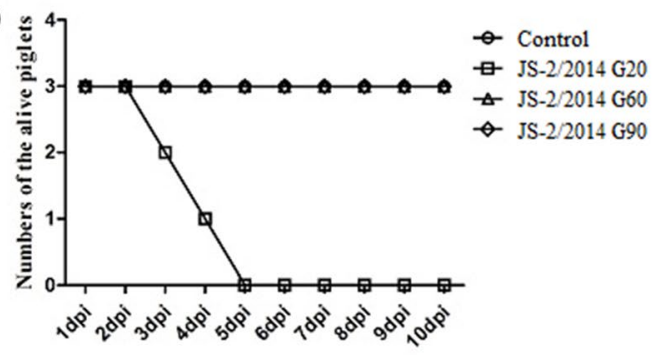

(d)

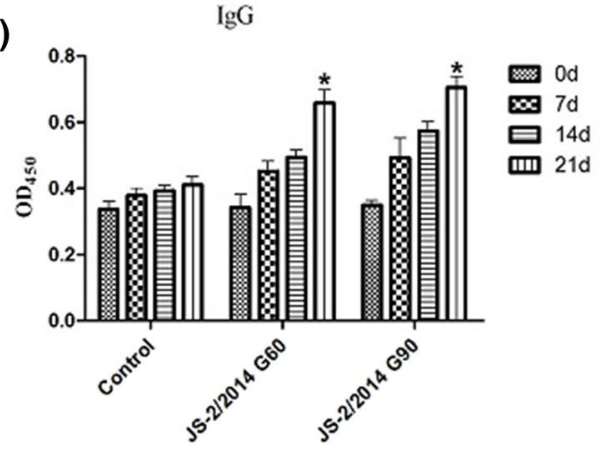

Fig. 4 Pathogenicity assays for a PEDV strain in piglets. Eight piglets were distributed into four groups and fed with $1 \mathrm{~mL}$ of PBS, JS-2/2014 G20, JS-2/2014 G60, or JS-2/2014 G90, respectively. Piglets fed with JS-2/2014 G20 died within 5 days and had severe intestinal lesions, while the other piglets were alive with no lesions in their

virus's escape from preexisting immunity. Thus, periodic vaccine updates may be required to ensure sufficient efficacy against emerging virus variants [28]. In the present study, our data revealed that there is little variation in the $S$ gene among the two PEDV isolates, and that they are most similar to the attenuated DR13 strain. To further explore the role of the $S$ gene in virulence, the JS-2/2014 strain was successively cultured, and $S$ gene variation during cultivation was analysed. Of note, we found that there were three amino acid mutations between JS-2/2014 G5 and JS-2/2014 G20, six between JS-2/2014 G20 and JS-2/2014 G50, and five between JS-2/2014 G50 and JS-2/2014 G90. It is worth noting that the amino acid variations between JS-2/2014 G5 and JS-2/2014 G50 were also identified in the DR13 attenuation process. This finding indicates that these missense mutations in the $S$ gene may be related to viral pathogenicity. Furthermore, animal experiments confirmed that piglets orally fed JS-2/2014 G5 $\left(10^{6} \mathrm{TCID}_{50}\right)$ had mild diarrhea and long-term excretion. On the other hand, piglets challenged

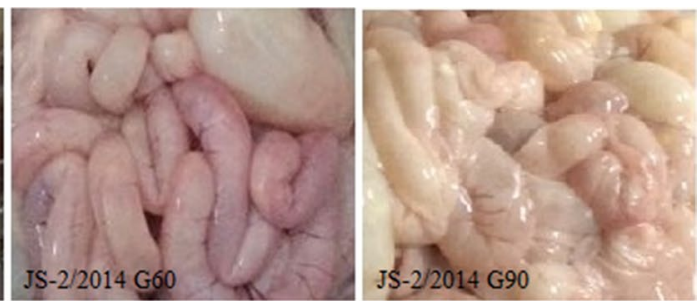

(c)

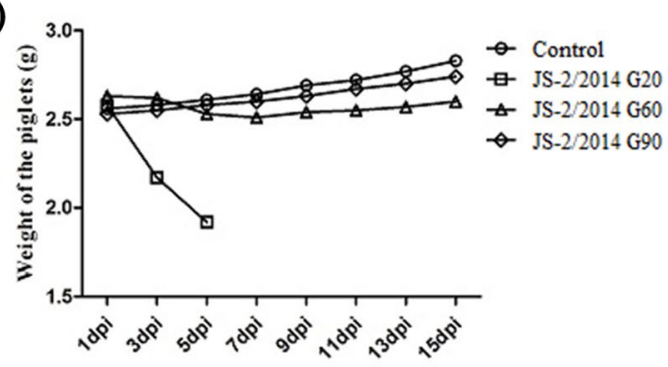

IgA

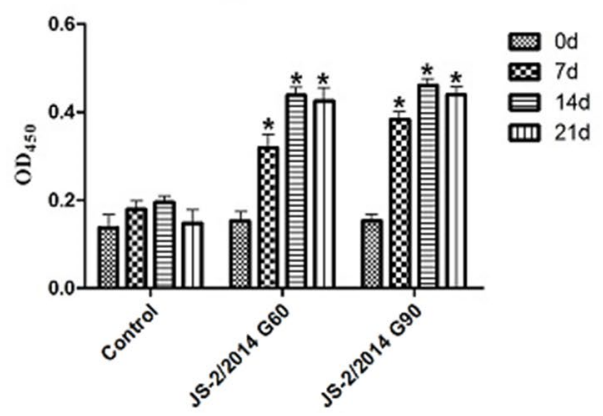

intestines (a and b). (c) Strains JS-2/2014 G60 and JS-2/2014 G90 had only a weak effect on piglet weight gain. Piglets fed with strain JS-2/2014 G90 manifested better weight gain than those fed with JS-2/2014 G60. (d) Oral immunization with JS-2/2014 G90 gave piglets high levels of $\operatorname{IgG}$ and IgA antibodies

with JS-2/2014 G90 $\left(10^{6} \mathrm{TCID}_{50}\right)$ appeared asymptomatic, with no virus detected in the feces and nasal fluid, indicating that G90 was attenuated and therefore is potentially safe for vaccine preparations. Moreover, recombination analysis confirmed that this strain has a high probability of being recombined with CV777 and DR13. Most recently, the $\mathrm{CH} / \mathrm{HNQX}-3 / 14$ strain was reported as a CV777 and DR13 strain recombinant with the highly virulent CHN/ ZMDZY/11 strain. Not surprisingly, this strain was detected on a swine farm that is located in the same province as the CHN/ZMDZY/11 strain; both CV777 and DR13 PEDV vaccines were used on this farm [29]. Therefore, it is possible that the virulence-enhanced CV777 and DR13 strains had been present in some areas, resulting in virus recombination.

In conclusion, this study investigated the epidemiology behind recent outbreaks of porcine diarrheal disease in China and identified two PEDV isolates from Shanghai and Jiangsu. Furthermore, we successfully obtained an attenuated PEDV strain by screening. The results should help our 
understanding of the evolution of PEDV and offer a suitable attenuated strain for vaccine preparation.

Funding This study was funded by the National Key Research and Development Program of China (No. 2018YFD0500100), Shanghai Agriculture Applied Technology Development Program of China (No. T20170111), and the Youth Talent Development Plan of Shanghai Municipal Agricultural System of China (No. 20160135).

\section{Compliance with ethical standards}

Conflict of interest There are no conflicts of interest associated with this article.

Ethical approval All applicable international, national, and/or institutional guidelines for the care and use of animals were followed. Furthermore, this article does not contain any experiments with human subjects or animals performed by any of the authors.

\section{References}

1. Jung K, Saif LJ (2015) Porcine epidemic diarrhea virus infection: etiology, epidemiology, pathogenesis and immunoprophylaxis. Vet J 204(2):134-143

2. Wang L, Byrum B, Zhang Y (2014) New variant of porcine epidemic diarrhea virus, United States. Emerg Infect Dis 20(5):917-919

3. Pasick J, Berhane Y, Ojkic D et al (2014) Investigation into the role of potentially contaminated feed as a source of the firstdetected outbreaks of porcine epidemic diarrhea in Canada. Transbound Emerg Dis 61(5):397-410

4. Stadler J, Zoels S, Fux R et al (2015) Emergence of porcine epidemic diarrhea virus in southern Germany. BMC Vet Res 11:142

5. Masuda T, Murakami S, Takahashi O et al (2015) New porcine epidemic diarrhoea virus variant with a large deletion in the spike gene identified in domestic pigs. Arch Virol 160(10):2565-2568

6. Li W, Li H, Liu Y et al (2012) New variants of porcine epidemic diarrhea virus, China, 2011. Emerg Infect Dis 18(8):1350-1353

7. Wang E, Guo D, Li C et al (2016) Molecular characterization of the ORF3 and $\mathrm{S} 1$ genes of porcine epidemic diarrhea virus non S-INDEL strains in seven regions of China, 2015. PLoS One 11(8):e0160561

8. Gao X, Li D, Zhao J et al (2016) Complete genome sequence of PEDV from an outbreak in a vaccinated farm in Shandong, China. Genome Announc 4(4):e00619-16

9. Li R, Qiao S, Yang Y et al (2016) Genome sequencing and analysis of a novel recombinant porcine epidemic diarrhea virus strain from Henan, China. Virus Genes 52(1):91-98

10. Wang K, Xie C, Zhang J et al (2016) The identification and characterization of two novel epitopes on the nucleocapsid protein of the porcine epidemic diarrhea virus. Sci Rep 6:39010

11. Sun D, Wang X, Wei S et al (2016) Epidemiology and vaccine of porcine epidemic diarrhea virus in China: a mini-review. J Vet Med Sci 78(3):355-363
12. Chen F, Zhu Y, Wu M et al (2015) Comparative genomic analysis of classical and variant virulent parental/attenuated strains of porcine epidemic diarrhea virus. Viruses 7(10):5525-5538

13. Jaru-Ampornpan P, Jengarn J, Wanitchang A et al (2017) Porcine epidemic diarrhea virus (PEDV) 3C-like protease-mediated nucleocapsid processing: a possible link to viral cell-culture adaptability. J Virol 91(2):e01660-16

14. Pan Y, Tian X, Li W et al (2012) Isolation and characterization of a variant porcine epidemic diarrhea virus in China. Virol J 9:195

15. Sun RQ, Leng ZM, Zhai SL et al (2014) Genetic variability and phylogeny of current Chinese porcine epidemic diarrhea virus strains based on spike, ORF3, and membrane genes. Sci World J 2014:208439

16. LaBarre DD, Lowy RJ (2011) Improvements in methods for calculating virus titer estimates from TCID50 and plaque assays. J Virol Methods 96(2):107-126

17. Song DS, Yang JS, Oh JS, Han JH, Park BK (2003) Differentiation of a vero cell adapted porcine epidemic diarrhea virus from Korean field strains by restriction fragment length polymorphism analysis of ORF 3. Vaccine 21:1833-1842

18. Ni JQ, Yuan L, Wang J et al (2017) Epidemiological survey on porcine diarrhea in swine breeding farms in China during 20132015. China Anim Health Insp 34(4):5-9

19. Chen J, Wang C, Shi H et al (2010) Molecular epidemiology of porcine epidemic diarrhea virus in China. Arch Virol 155(9):1471-1476

20. Trudeau MP, Verma H, Sampedro F et al (2017) Environmental persistence of porcine coronaviruses in feed and feed ingredients. PLoS One 12(5):e0178094

21. Chang TC, Chen JF, Feng L et al (2016) Epidemiological survey of porcine epidemic diarrhea virus in 2014. Chin J Prev Vet Med 38(4):335-338

22. Hu XY, Zhang SX, Jin ZQ et al (2016) The epidemiological investigation on viral diarrhea of piglets in Guizhou region. Chin J Prev Vet Med 38(7):542-545

23. Zhu WX, Guo HY, Chen LG et al (2012) Isolation and identification of porcine epidemic diarrhea virus strain HBMC2012 and its pathogenicity in piglet. Chin J Prev Vet Med 38(12):934-938

24. Li W, van Kuppeveld FJ, He Q et al (2016) Cellular entry of the porcine epidemic diarrhea virus. Virus Res 226:117-127

25. Park SJ, Moon HJ, Luo Y et al (2008) Cloning and further sequence analysis of the ORF3 gene of wild- and attenuated-type porcine epidemic diarrhea viruses. Virus Genes 36(1):95-104

26. McGoldrick A, Lowings JP, Paton DJ (1999) Characterisation of a recent virulent transmissible gastroenteritis virus from Britain with a deleted ORF 3a. Arch Virol 144(4):763-770

27. Park SJ, Song DS, Ha GW, Park B (2007) Cloning and further sequence analysis of the spike gene of attenuated porcine epidemic diarrhea virus DR13. Virus Genes 35(1):55-64

28. Lin CM, Saif LJ, Marthaler D, Wang Q (2016) Evolution, antigenicity and pathogenicity of global porcine epidemic diarrhea virus strains. Virus Res 226:20-39

29. Li R, Qian S, Yang Y et al (2016) Genome sequencing and analysis of a novel recombinant porcine epidemic diarrhea virus strain from Henan, China. Virus Genes 52(1):91-98 